\title{
Adverse Events and Their Contributors Among Older Adults During Skilled Nursing Stays for Rehabilitation: A Scoping Review
}

\author{
Udoka Okpalauwaekwe (iD) \\ Huey-Ming Tzeng (iD ${ }^{2}$ \\ 'University of Saskatchewan, College of \\ Medicine, Saskatoon, Saskatchewan, \\ Canada; ${ }^{2}$ The University of Texas Medical \\ Branch at Galveston, School of Nursing, \\ Galveston, TX, USA
}

Purpose: To identify factors that contribute to adverse events among older adults during short stays at skilled nursing facilities (SNFs) for rehabilitation (ie, up to 100 resident days). Adults aged 65 years and older are at serious risk for adverse events throughout their continuum of care. Over $33 \%$ of older adults admitted to SNFs experienced an adverse event (eg, falls) within the first 35 days of their stay.

Design: A scoping review.

Setting and Participants: Older adults admitted for short stays in SNFs.

Methods: Eligibility criteria were peer-reviewed original articles published between 1 January 2015 and 30 May 2021, written in English, and containing any of the following key terms and synonyms: "skilled nursing facilities", "adverse events", and "older adults". These terms were searched in PubMed, MEDLINE, CINAHL, EBSCOHost, and the ProQuest Nursing and Allied Health Database. We summarized the findings using the Joanna Briggs Institute and PRISMA-ScR reporting guidelines. We also used the CapabilityOpportunity-Motivation-Behavior (COM-B) model of health behavioral change as a framework to guide the content, thematic, and descriptive analyses of the results.

Results: Eleven articles were included in this scoping review. Intrinsic and extrinsic contributors to adverse events (ie, falls, medication errors, pressure ulcers, and acute infections) varied for each COM-B domain. The most frequently mentioned capacity-related intrinsic contributors to adverse events were frailty and reduced muscle strength due to advancing age. Inappropriate medication usage and polypharmacy were the most common capacityrelated extrinsic factors. Opportunity-related extrinsic factors contributing to adverse events included environmental hazards, poor communication among SNF staff, lack of individualized resident safety plans, and overall poor care quality owing to racial bias and organizational and administrative issues.

Conclusion: These findings shed light on areas that warrant further research and may aid in developing interventional strategies for adverse events during short SNF stays.

Keywords: older adults, adverse events, skilled nursing facilities, rehabilitation, short stays, risk factors

\section{Introduction}

The US Department of Health and Human Services and the Agency for Healthcare Research and Quality (AHRQ) acknowledge that adults aged 65 years and older are at serious risk for adverse events throughout their continuum of care. ${ }^{1,2}$ In 2014, the Office of the Inspector General reported that more than $33 \%$ of skilled nursing facility (SNF) beneficiaries experienced an adverse event or temporary-harm event 
within the first 35 days of their stay. ${ }^{1}$ In this context, "adverse event" describes any medical care-related harm or injury, including the failure to provide needed care. ${ }^{1-3}$ Adverse events, such as falls, falls with injuries, medical errors, and substandard care, are common among older adults. Adverse events can result in preventable harms (eg, healthcare-acquired infections, adverse drug events, malnutrition, pressure ulcers, and medication errors) during care delivery to older adults. . $^{1,3,4}$

Adverse events among older adults occur most often in the immediate post-hospitalization period, ${ }^{1,3}$ owing to several factors that can be classified as either intrinsic or extrinsic. ${ }^{5,6}$ Intrinsic factors are individual-related factors such as age, fear of falling, cognition, health condition, and medications. ${ }^{6,7}$ Extrinsic factors are external to the individual, including influences from outside the individual, such as the environment and social and cultural influences. ${ }^{6,7}$ Although older adults in SNFs are considered the most vulnerable to adverse events, ${ }^{1}$ insufficient evidence exists to support the degree of vulnerability or determine the reasons for this pattern. Additionally, because more research focuses on older adults in acute hospital settings compared with SNFs which have been shown in research to admit more acute older adults for rehabilitative care; ${ }^{3,8-10}$ it is therefore plausible that the frequency of adverse events occurrence in SNFs may be higher than recorded in the literature.

According to the United States Center for Disease Control and Prevention, 14\% of adults aged 65 years and over experience a repeat fall within 1-month posthospitalization or visit the emergency room for a fallrelated issue. ${ }^{11}$ Milat et al reported that $40 \%$ of older adults fall within 6 months post-discharge and thus require continued assistance for up to 6 months post-discharge. ${ }^{12}$ In an epidemiological study, Kapoor et al found that $40 \%$ of older adults admitted to long-term care facilities posthospitalization experience adverse events such as falls and infections within 45 days after admission. ${ }^{3}$ Given the frequency of adverse events in SNFs, we explored the risk factors associated with adverse events in SNFs and analyzed the relationships between these risk factors and adverse events among older adults admitted for short stays in SNFs. This scoping review explores the evidence in the literature reporting extrinsic and intrinsic risk factors that contribute to adverse events among older adults admitted to SNFs for short-stay rehabilitation. In this study, we defined short stays as SNF stays up to 100 resident days for rehabilitative care.

\section{Study Rationale and Objectives}

We conducted a scoping review of the peer-reviewed published journal literature to (1) identify the extent, range, and quantity of evidence available regarding short-stay rehabilitative care for older adults in SNFs (up to 100 days) and factors that contribute to related adverse events (ie, fall/fall injuries, pressure ulcers, medication errors, and acute infections); (2) map and describe these factors (intrinsic and extrinsic) using the Capability-Opportunity-MotivationBehavior (COM-B) model of health behavioral changes ${ }^{13}$ as a framework, and (3) determine existing gaps in the research. The COM-B model categorizes behavioral change strategies into three groups: capability (eg, physical or psychological-related changes), opportunity (eg, physical opportunities afforded by the environment or social opportunities afforded by cultural milieu), and motivation (eg, reflective-related or planned changes, as well as automaticrelated or impulse changes). This model could support intervention designs and improve intervention evaluation and theory development. ${ }^{13}$ The overarching research question was What are the intrinsic and extrinsic factors contributing to adverse events (ie, falls/fall injuries, pressure ulcers, medication errors, and acute infections) among older adults during short SNF stays for rehabilitation?

\section{Materials and Methods}

We systematically searched and reviewed articles within the disciplines of nursing, medicine, and geriatrics, using the Joanna Briggs Institute (JBI) guideline for scoping reviews ${ }^{14}$ based on the Arksey and O'Malley methodological framework. ${ }^{15}$ Our search strategy focused on both primary and secondary sources (including systematic reviews) that described adverse events among older adult residents in nursing homes. The major adverse events we focused on were falls, falls with injuries, pressure ulcers, medication errors, and acute infections. We followed the guidelines of the Preferred Reporting Items for Systematic Reviews and Meta-Analyses Extension for Scoping Reviews (PRISMA-SCR). ${ }^{16}$

\section{Protocol and Registration}

No published or registered protocol was in place before the study commenced.

\section{Eligibility Criteria}

Articles were included if they met the following criteria: 1) were peer-reviewed, 2) reported associations, predictors, 
correlations, associations, and/or relationships between falls/fall injuries (or other adverse events such as medication errors, infections, and pressure ulcers) and older adults' characteristics, 3) included older adults admitted to SNFs for short-stay rehabilitative care, and 4) were published between 01 January 2015 and 30 May 2021 and were written in English.

\section{Information Sources and Search Strategy}

We worked with a librarian to identify three conceptual groups of combined and individually adapted terms for each database-specific search. These terms included "skilled nursing facilities" (including synonyms), "adverse events" (including falls, pressure ulcers, medication errors, and acute infections as synonyms), and older adults (including synonyms). Table 1 shows the search syntax generated and modified to each electronic database. Studies were identified by searching these databases and hand-searching reference lists of the included articles.

The following databases were searched: Medline-Ovid (1 January 2015 to 30 May 2021), PubMed (1 January 2015 to 30 May 2021), Cumulative Index to Nursing and Allied Health Literature (CINAHL; 1 January 2015 to 30 May 2021), EBSCOHost (1 January 2015 to 30 May 2021), and the ProQuest Nursing and Allied Health Database (1 January 2015 to 30 May 2021). The initial search was performed between 10 November 2020 and 15 December 2020, with two updated searches on 30 January 2021 and 30 May 2021. We hand-searched references of the included articles and used the snowballing method to identify relevant papers. No gray literature was included in the complimentary searches.

\section{Selection of Evidence Sources}

Both authors screened the citations and articles against the preset inclusion criteria described in the section titled "Eligibility criteria". We applied two iterations of the same approach to identify articles for inclusion. First, we screened the titles and abstracts of all articles collated from the library databases and removed the duplicates. Next, we retrieved the full texts of the remaining articles and reviewed them for relevance (according to the study's research question), assigning a score of either 0 (not relevant to the research question) or 1 (relevant to the research question). We then discussed conflicts and discrepancies between inter-rater scores and resolved them by discussion. The overall inter-rater reliability Kappa score was
Table I Keyword Search Syntax and Search Strategy for the Five Library Databases (PubMed, MEDLINE, CINAHL, EBSCOHost, and ProQuest Nursing and Allied Health Database)

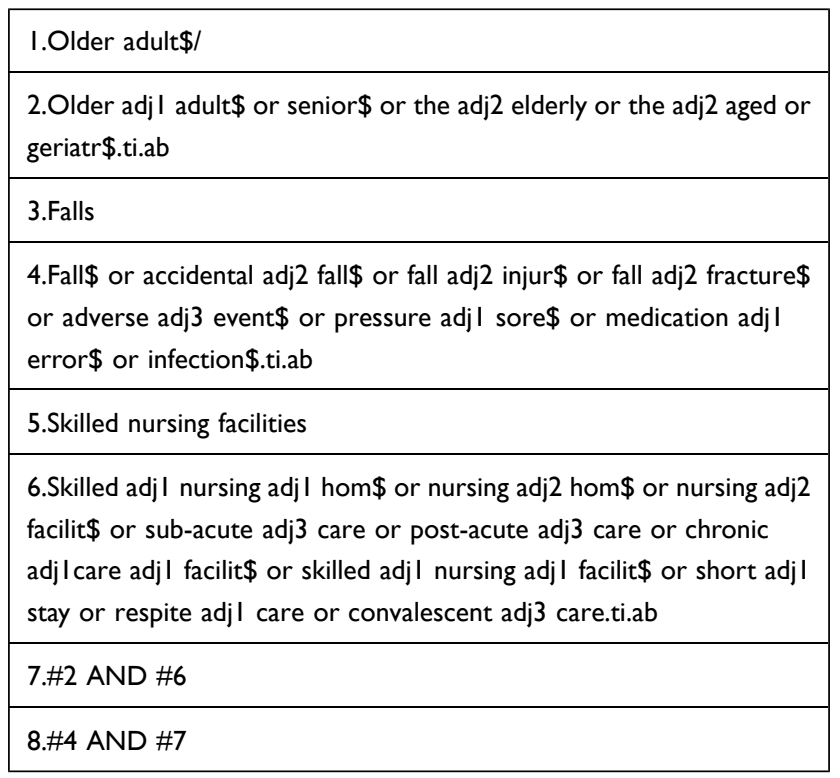

Notes: The search items in Table I uses truncation (eg, \$) at the end of a word to facilitate finding the word in its several endings or spelling styles. For example, geriatr $\$$ would find geriatrics, geriatric, geriatrician, etc., without entering them manually. Same for facility $\$$ for facility, or facilities where necessary. Adj operators in our table were used to find two or more terms adjacent to each other. For example, adjl would find terms right next to each other, adj2 would find terms in any other with one word between them, and adj 3 will find terms in any order with one to two words between them. For instance, older Adjl adults would find the word older adults, and nursing adj2 home will find the word nursing home or nursing "care" home (assuming the word care was included in between them). These codes were put together with the help of a professional librarian to ensure a comprehensive search of keywords and Mesh terms.

0.846 (standard error $=0.147, \mathrm{p}=0.001$ ). All citations were imported or manually entered using the reference manager, Endnote X9. ${ }^{17}$

\section{Data Charting and Data Items}

We extracted the following preidentified data from the final selected articles: author names, title and date of publication, study type, design, data collection methods, study setting and country, adverse event types, length of stay in SNF, intrinsic and extrinsic factors that contributed to each adverse event among older adults during rehabilitation or respite care, study gaps, and lessons learned. For each selected study, both authors extracted and coded the data for intrinsic and extrinsic factors as described from each included article, using the COM-B model of health behavioral change as a framework. ${ }^{13}$ Using this framework, we characterized the intrinsic and extrinsic factors related to specified adverse events among older adults during short stays in SNFs to determine behavioral strategies that could 
mitigate these factors. ${ }^{13}$ All data were compiled into a spreadsheet using Microsoft Excel 2016. ${ }^{18}$

\section{Critical Appraisal of Individual Evidence Sources}

We appraised each included article's characteristics and methodological quality using the JBI critical appraisal tool for quantitative studies (eg, randomized clinical, prospective, retrospective, and cross-sectional studies). ${ }^{19}$ The JBI critical appraisal tool enables evaluating the rigor, trustworthiness, relevance, and potential for bias in study designs, conduct, and analysis. ${ }^{19}$ Please see the Supplementary Materials Tables $1-\underline{4}$ for the critical appraisal data of the included studies using the JBI critical appraisal tools for study designs.

\section{Synthesis of Results}

We used the COM-B model of health behavioral change to analyze the data and descriptive results of this scoping review. The COM-B model categorizes behavioral change strategies into one of three groups: capability, opportunity, or motivation. ${ }^{13}$ Using this model may help design interventions and improve intervention evaluation and theory development. ${ }^{13}$ We characterized the behavioral change strategies related to preventing the predefined adverse events among older adults during short SNF stays for rehabilitation. Both authors met weekly via the internet to review codes and themes from the data analyses. Conflicting themes were resolved by discussion.

\section{Results}

\section{Selection of Evidence Sources}

We identified 325 articles from five databases $(n=311)$ and by hand-searching/snowballing $(\mathrm{n}=14)$. Of these 325 articles, 78 were duplicates, resulting in 247 articles to be further screened. After screening the title and abstracts, we excluded 121 articles, leaving 126 articles for which we retrieved and assessed the full texts for eligibility. After screening the full texts, we excluded 115 articles, leaving 11 articles for data extraction and final review. Full-text articles were excluded from final screening for the following reasons: 1) no indication of a subacute or post-acute rehabilitation stay in a nursing home or SNF, 2) no indication of adverse events during the nursing home or SNF stay, 3) article was not written in English, or 4) article was not an original study (ie, discussion paper, commentary, essay, or dissertation) (Figure 1).

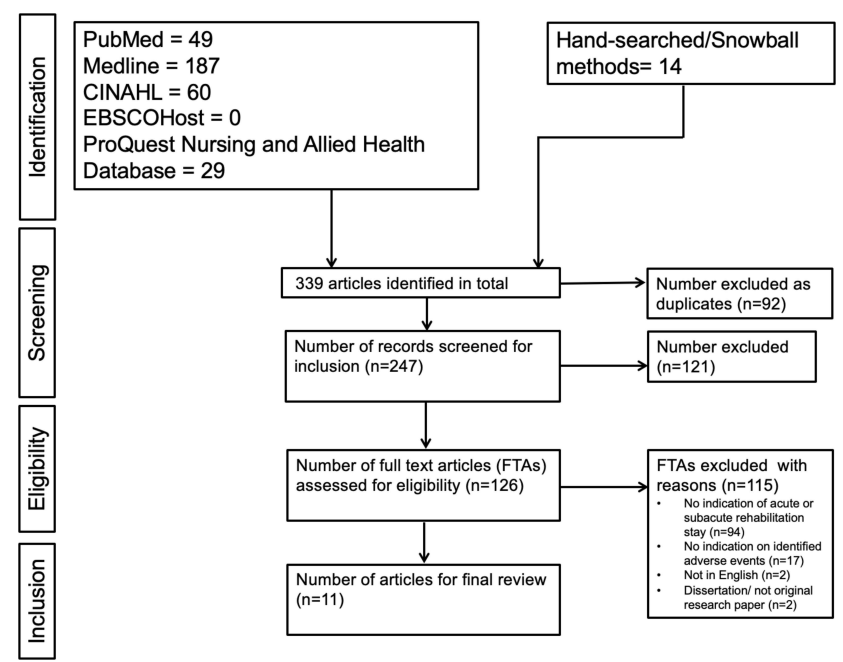

Figure I PRISMA flowchart showing the article selection process for the scoping review.

Note: Adapted from Tricco AC, Lillie E, Zarin W, et al. PRISMA extension for scoping reviews (PRISMA-ScR): checklist and explanation. Ann Intern Med. 20I8;169 (7):467-473. doi:10.7326/MI8-0850. ${ }^{16}$

\section{Characteristics of Evidence Sources}

Table 2 summarizes the general and methodological characteristics of the 11 reviewed articles. Of these, three were published in 2015, two in 2016, three in 2017, two in 2018, and one in 2019. We found no articles published in 2020 or 2021 . Ten $(90.9 \%)$ were quantitative studies, and one (9.9\%) used a mixed method. Four (36.4\%) used a cross-sectional design, three $(27.3 \%)$ were prospective, and four (36.4\%) were retrospective. Six studies (54.5\%) were conducted in the United States, and three were conducted in the Netherlands. One study mentioned multiple adverse events. Falls and fall injuries were the most common adverse events (10 studies, 90.9\%), followed by two studies reporting medication errors, one reporting pressure ulcers, and one reporting acute infections. Six (54.5\%) of the included studies indicated length of stay; five (45.5\%) did not. We contacted the corresponding authors of these studies to confirm the participants' length of stay. Table 3 summarizes the characteristics of the included studies.

\section{Synthesis of Results}

Table 4 describes the intrinsic factors (left column) and extrinsic factors (right column) that contributed to adverse events among older adults during short SNF stays for rehabilitation as reported in all included studies. We coded them with identifiers corresponding to the COM-B framework ${ }^{13}$ where "C" indicates capability, "O" indicates 
Table 2 General and Methodological Characteristics of the II Included Quantitative Articles Published Between 2015 and 202 I

\begin{tabular}{|c|c|c|}
\hline Publication Year & n (\%) & Article Citations \\
\hline 2015 & $3(27.3)$ & {$[21,24,27]$} \\
\hline 2016 & $2(18.2)$ & {$[23,25]$} \\
\hline 2017 & $3(27.3)$ & {$[22,26,29]$} \\
\hline 2018 & $2(18.2)$ & {$[6,28]$} \\
\hline 2019 & I (9.1) & {$[20]$} \\
\hline Study type & n (\%) & Article citations \\
\hline Quantitative & $10(90.9)$ & {$[6,20-24,26-29]$} \\
\hline Mixed method (including both qualitative and quantitative data collections) & I (9.1) & {$[25]$} \\
\hline Study design & n (\%) & Article citations \\
\hline Cross-sectional & $4(36.4)$ & {$[21,22,25,29]$} \\
\hline Prospective & $3(27.3)$ & {$[6,26,27]$} \\
\hline Retrospective & $4(36.4)$ & {$[20,23,24,28]$} \\
\hline Data collection methods & n (\%) & Article citations \\
\hline Structured survey questionnaires & $6(54.5)$ & {$[6,21,22,26-28]$} \\
\hline Semi-structured questionnaire & I (9.1) & {$[25]$} \\
\hline Retrospective data collection & $4(36.4)$ & {$[20,23,24,29]$} \\
\hline Country & n (\%) & Article citations \\
\hline Canada & I (9.1) & {$[28]$} \\
\hline Norway & I (9.1) & [29] \\
\hline The Netherlands & $3(27.3)$ & {$[23,26,27]$} \\
\hline United States & $6(54.5)$ & {$[6,20-22,24,25]$} \\
\hline Adverse event type & n (\%) & Article citations \\
\hline Falls/fall injuries & $10(90.9)$ & {$[6,20-28]$} \\
\hline Pressure ulcers & I (9.1) & {$[25]$} \\
\hline Medication errors & $2(18.2)$ & {$[21,29]$} \\
\hline Acute infections & I (9.1) & [25] \\
\hline Length of stay in the skilled nursing facilities & n (\%) & Article citations \\
\hline Up to 100 days (short stay) & $4(36.4)$ & {$[20,21,25,28]$} \\
\hline More than 100 days (long days) & $0(0.0)$ & - \\
\hline Variable (mix of short and long stays) & $2(18.2)$ & {$[23,29]$} \\
\hline Not specified & $5(45.5)$ & {$[6,22,24,26,27]$} \\
\hline
\end{tabular}

opportunity and " $\mathrm{M}$ " indicates motivation for the respective intrinsic (coded with the prefix "In") or extrinsic (coded with the prefix "Ex") factors for each theme. Subthemes were similarly categorized under major themes using the same coding strategy.

\section{Intrinsic Factors}

We identified the following themes as capacity-related intrinsic factors: a) frailty and reduced muscle strength due to advancing age, ${ }^{20-25}$ b) sex,,$^{6,23,25}$ c) fall history, $^{20-22,24,25}$ d) cognitive impairment ${ }^{20,21,24,26,27}$ and e) comorbidities ${ }^{24-27}$ (Table 4). These capacityrelated intrinsic factors were reported to contribute to falls, pressure ulcers, and medication errors. The most frequently mentioned capacity-related contributors to adverse events were frailty and reduced muscle strength due to advancing age (6 articles, 54.5\%). ${ }^{20-25}$ Fall history $^{20-22,24,25}$ and cognitive impairment ${ }^{20,21,24,26,27}$ were the next most common contributors (5 articles each, 45.5\% each) (fall and fall injuries, specifically) during short stays. The most recurring subthemes implicated as contributors to falls, medication errors, and 


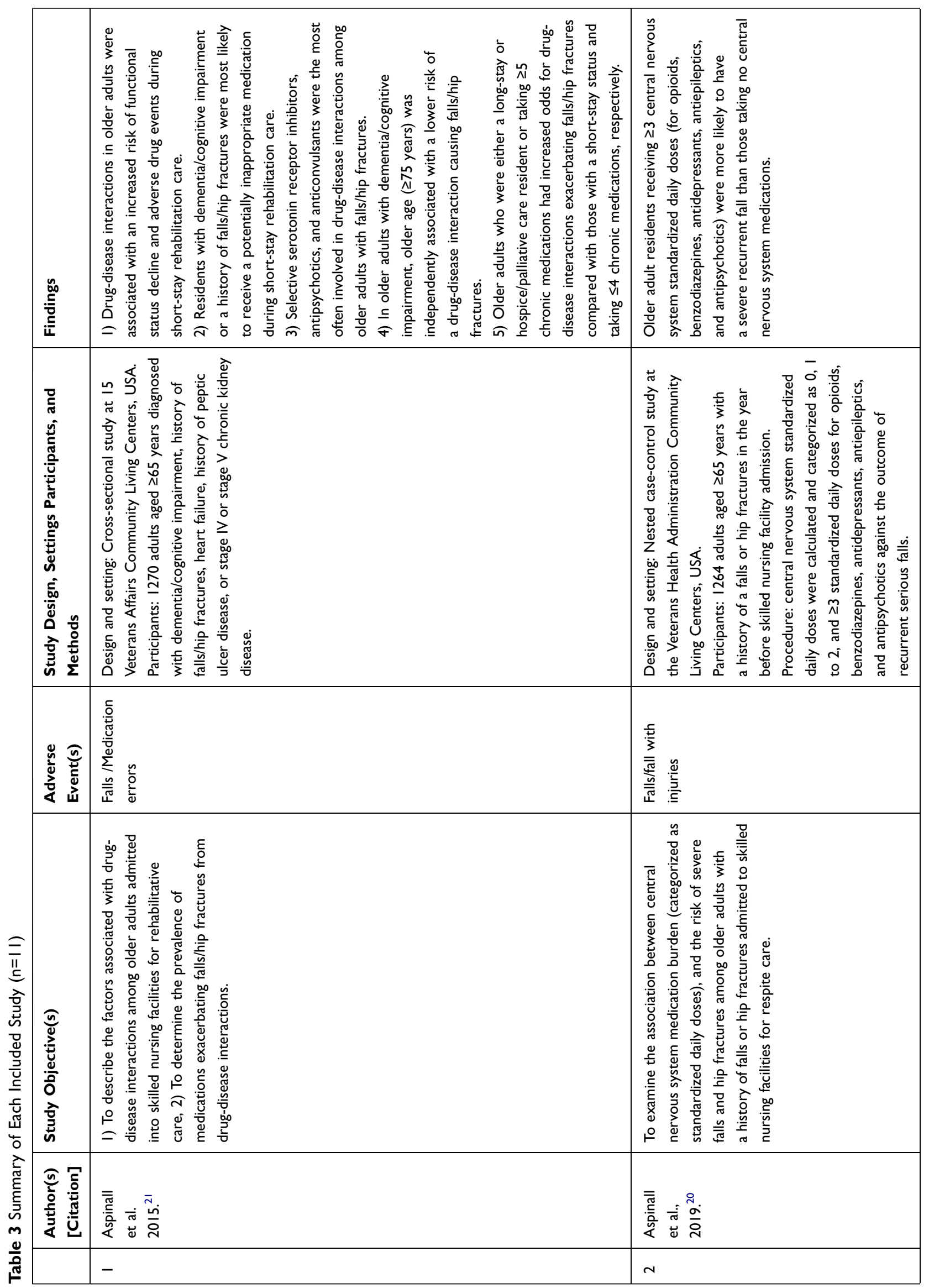




\begin{tabular}{|c|c|c|}
\hline 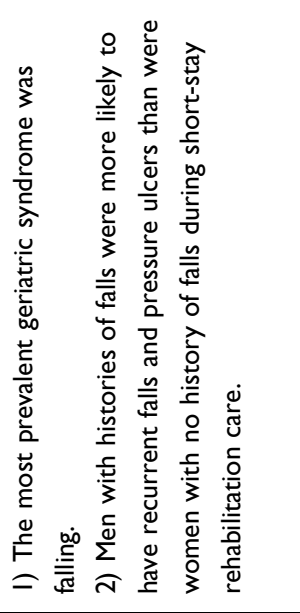 & 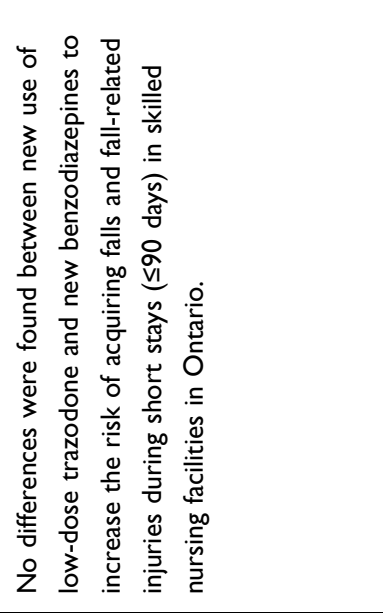 & 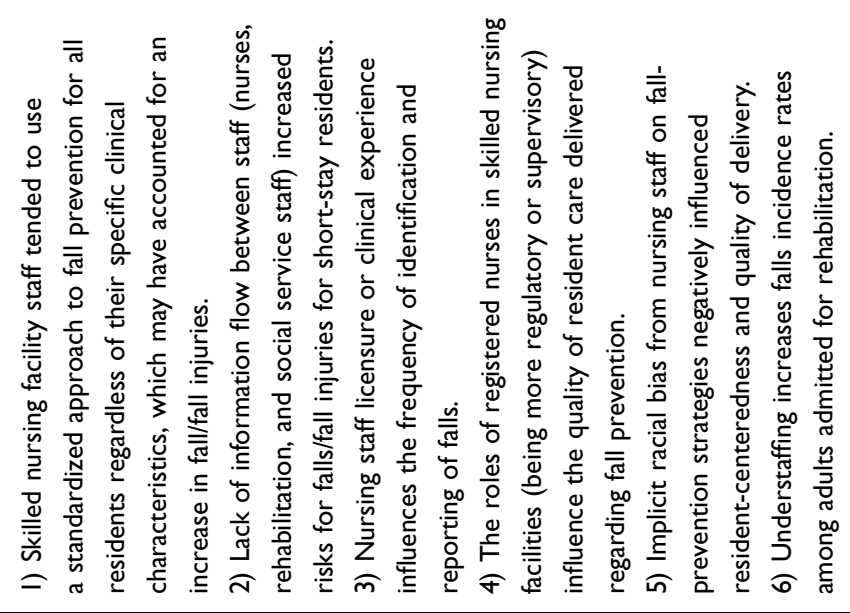 \\
\hline 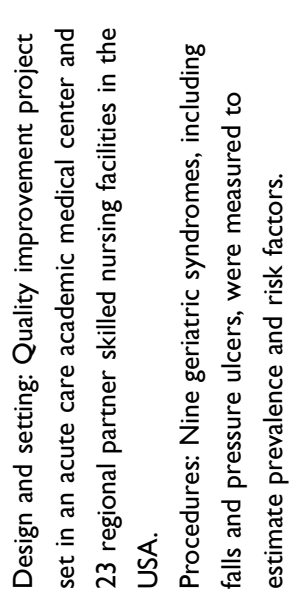 & 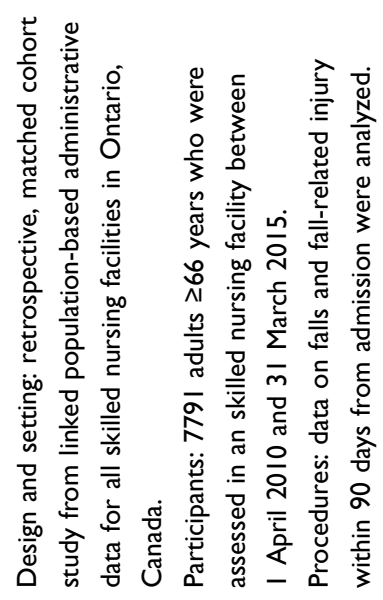 & 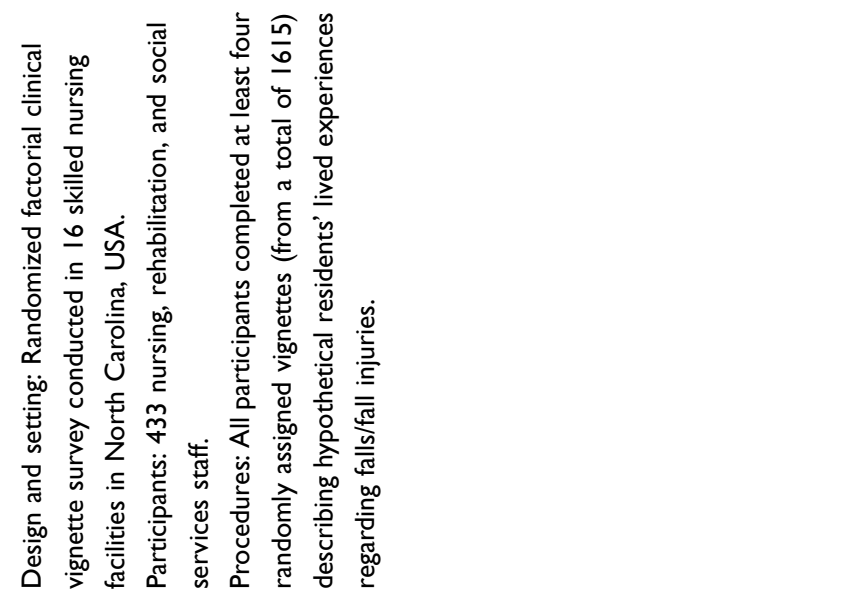 \\
\hline 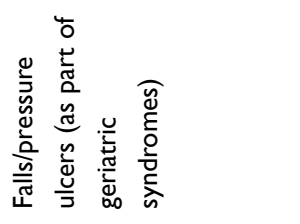 & 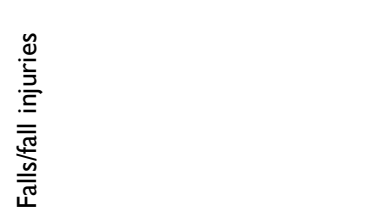 & 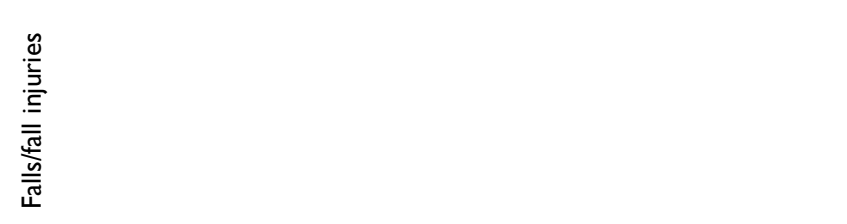 \\
\hline 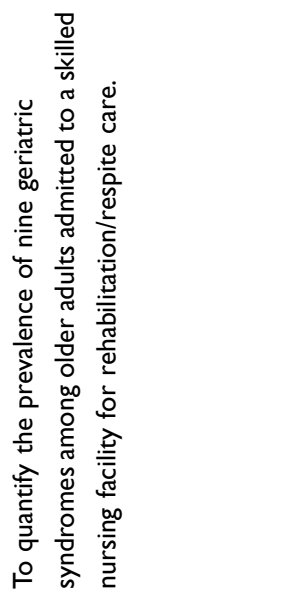 & 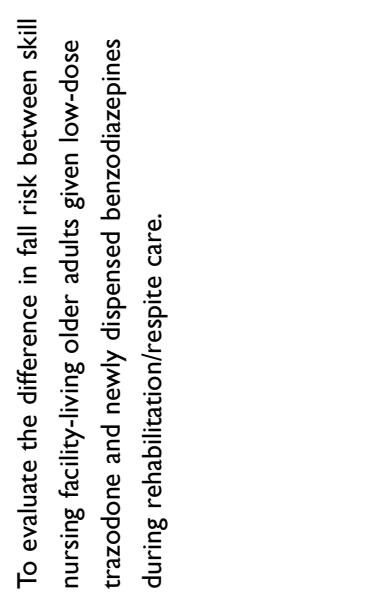 & 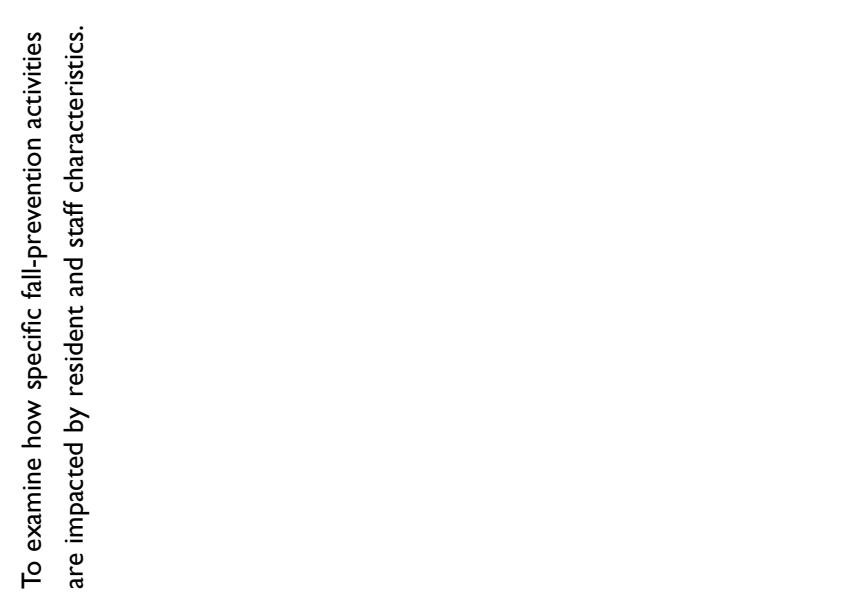 \\
\hline 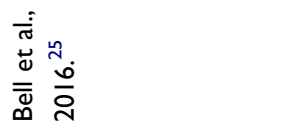 & 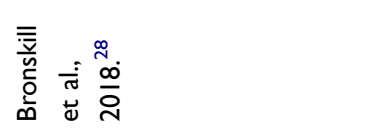 & 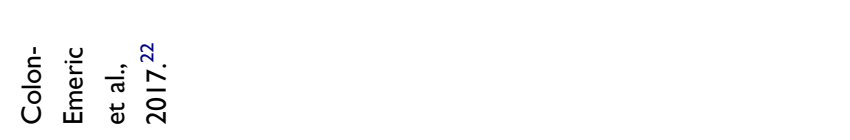 \\
\hline$m$ & $\sigma$ & in \\
\hline
\end{tabular}




\begin{tabular}{|c|c|c|c|c|c|}
\hline 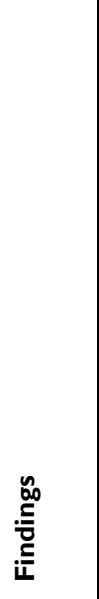 & 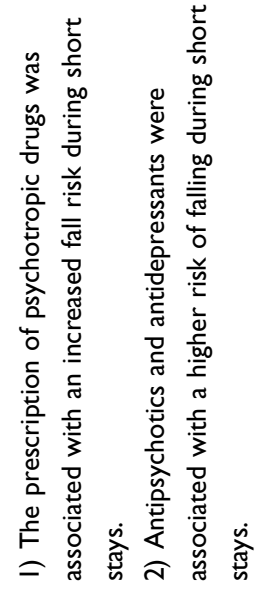 & 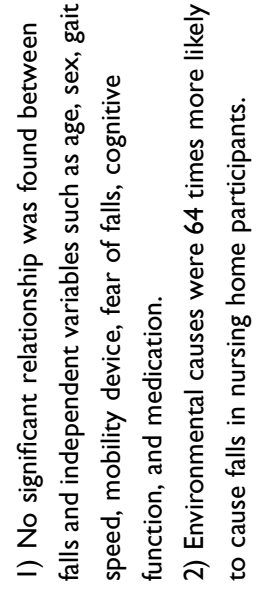 & 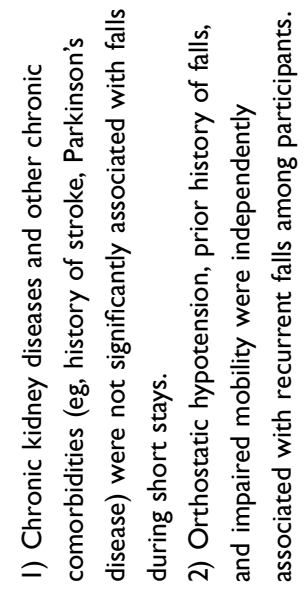 & 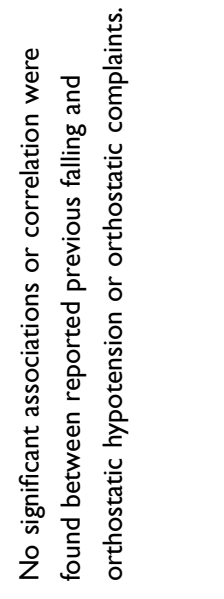 & 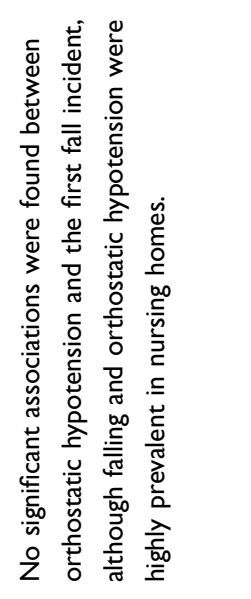 \\
\hline 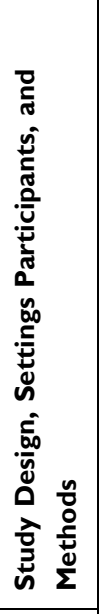 & 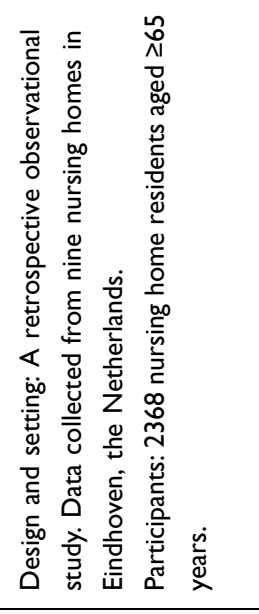 & 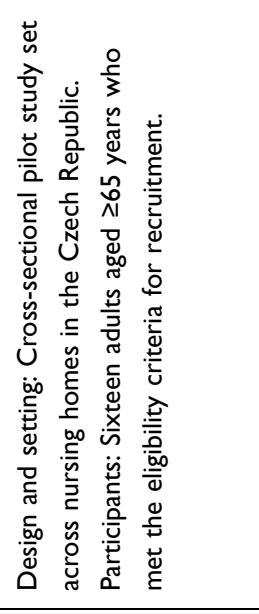 & 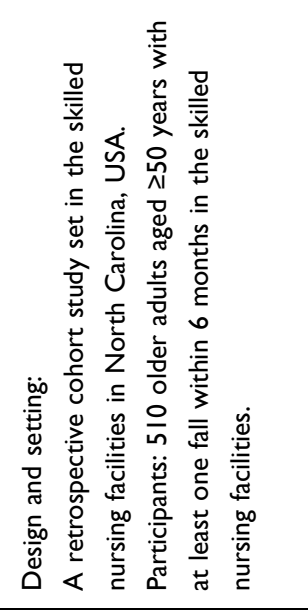 & 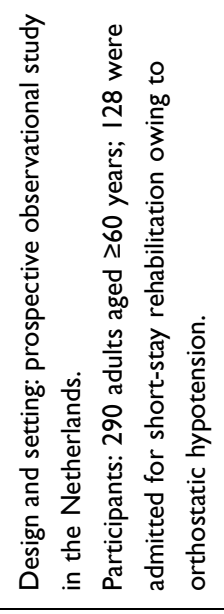 & 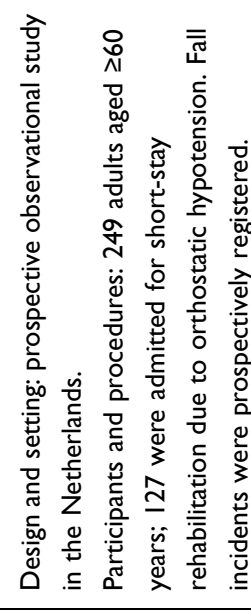 \\
\hline 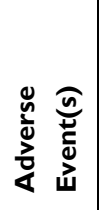 & 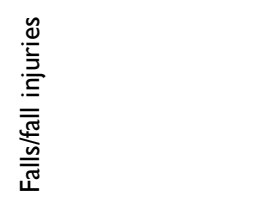 & 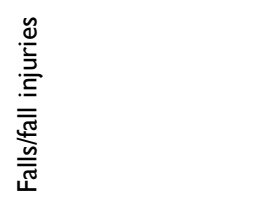 & 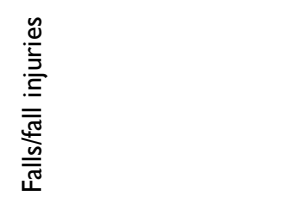 & 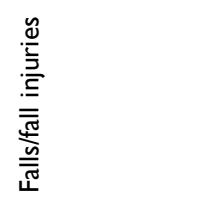 & 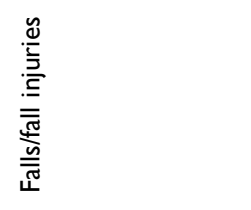 \\
\hline 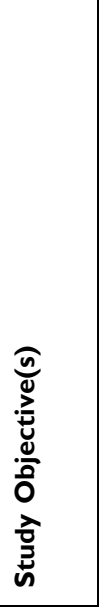 & 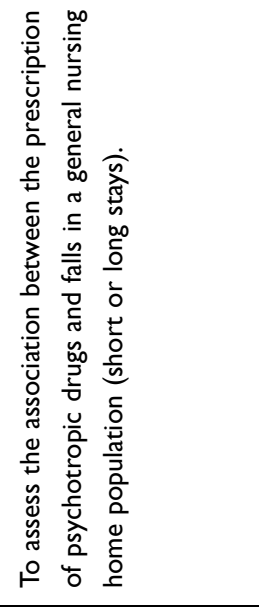 & 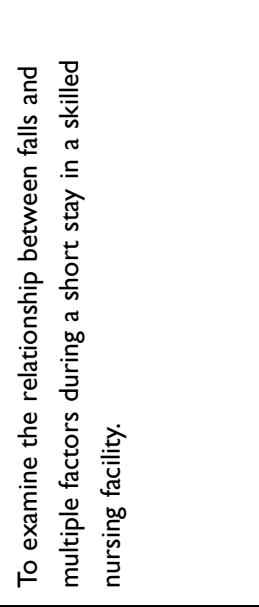 & 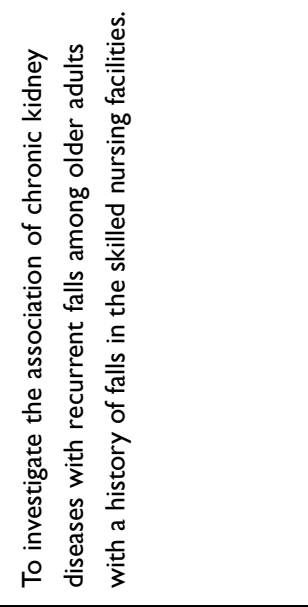 & 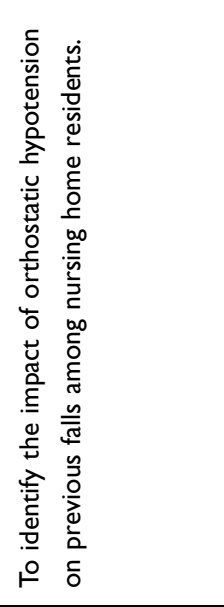 & 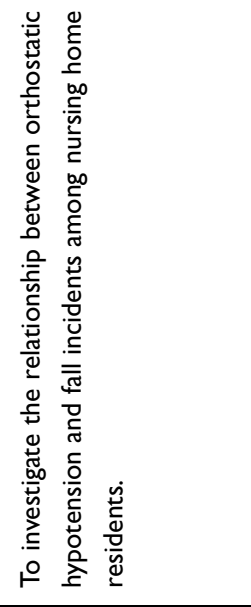 \\
\hline 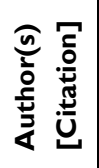 & 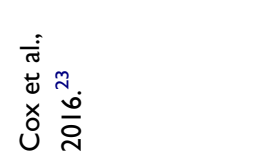 & 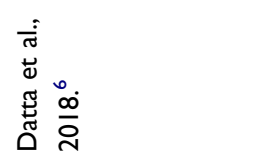 & 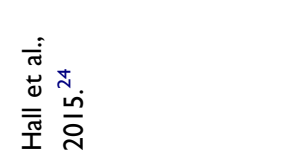 & 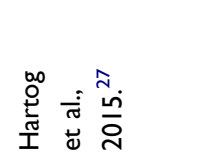 & 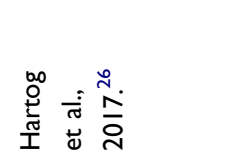 \\
\hline & 0 & $\wedge$ & $\infty$ & $\sigma$ & 으 \\
\hline
\end{tabular}



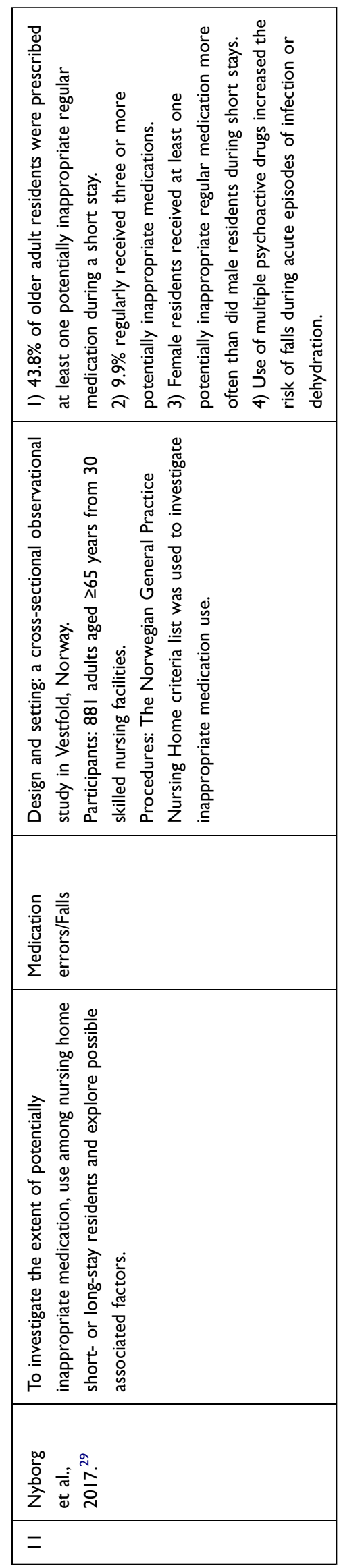

pressure ulcers were ambulation difficulties (eg, being bedbound or using supports such as a cane, walker, or wheelchair) (5 articles, $45.5 \%) .{ }^{20-22,24,25}$ We identified no opportunity-related or motivation-related intrinsic factors contributing to adverse events in older adults during short SNF stays (Table 4).

\section{Extrinsic Factors}

We identified and categorized inappropriate medications and issues related to polypharmacy as capacity-related extrinsic factors contributing to adverse events during short SNF stays. These capacity-related extrinsic factors were reported to contribute to falls and medication errors. Inappropriate administration of central nervous system (CNS) medications (eg, selective serotonin receptor inhibitors, opioids, benzodiazepines, antidepressants, anticonvulsants, and antipsychotics) was the most commonly identified capacity-related (extrinsic) subtheme contributing to most fall and fall injury incidences among older adults during short stays in SNFs (4 articles, 36.4\%). ${ }^{20,21,23,28}$ Polypharmacy (taking $\geq 3$ medications) was also a capacityrelated extrinsic factor contributing to medication errors and drug-disease interactions leading to fall and fall injuries during short stays (3 articles, 27.3\%). ${ }^{21,23,29}$

Six themes were identified and categorized as opportunity-related extrinsic factors contributing to falls and fall injuries during SNF short stays. ${ }^{22}$ The themes included 1) environmental safety factors ${ }^{6}$ (eg, clutter, lack of stair railings, lack of grab bars in the bathroom, poor lighting, loose rugs or other tripping hazards), 2) poor communication among SNF staff, 3) lack of older adult engagement in prevention care plans during short SNF stays, 4) lack of an individualized adverse event prevention plan, 5) organizational and administrative issues (eg, staff shortages, lack of delineation of roles for staff), and 6) care disparity related to racial bias (Table 4). ${ }^{22}$ We identified no motivationrelated extrinsic factors contributing to adverse events in older adults during short SNF stays.

\section{Discussion}

In this scoping review, we synthesized peer-reviewed evidence on intrinsic and extrinsic factors that contribute to adverse events (ie, falls, medication errors, pressure ulcers, and acute infections) among older adults admitted to SNFs for rehabilitation. We used the COM-B framework ${ }^{13}$ to categorize the intrinsic and extrinsic factors identified in 11 articles to determine behavioral modifications and gaps to address in future research. Grouping the intrinsic and 


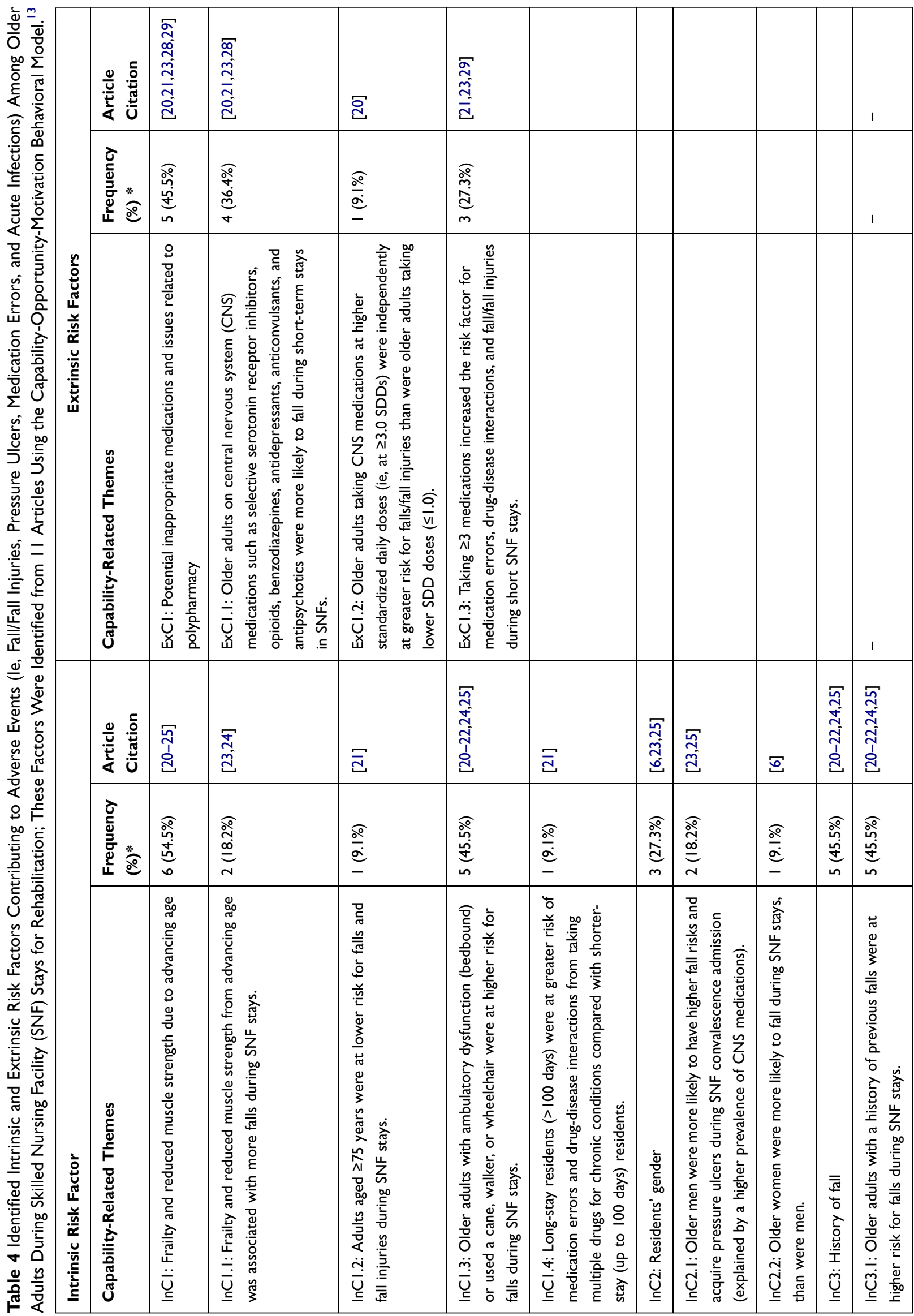




\begin{tabular}{|c|c|c|c|c|c|c|c|c|c|c|}
\hline & 1 & & 1 & 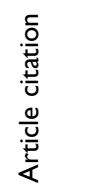 & $\bar{e}$ & $\Xi$ & $\mathbb{\Xi}$ & $\mathbb{Z}$ & $\mathbb{Z}$ & $\widetilde{\Xi}$ \\
\hline & 1 & & 1 & 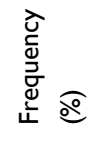 & $\frac{\widehat{\circ}}{\stackrel{\circ}{\sigma}}$ & $\frac{\widehat{ఠ}}{\stackrel{9}{a}}$ & $\begin{array}{l}\frac{\nwarrow}{\circ} \\
\stackrel{\circ}{-}\end{array}$ & $\frac{\widehat{o}}{\frac{\sigma}{\sigma}}$ & $\frac{\widehat{ఠ}}{\stackrel{0}{a}}$ & $\frac{\widehat{o}}{\stackrel{\circ}{\sigma}}$ \\
\hline & 1 & & 1 & 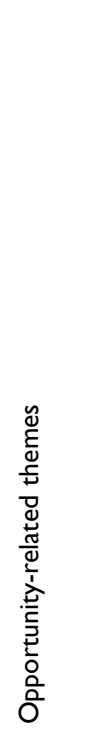 & 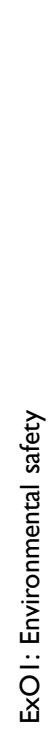 & 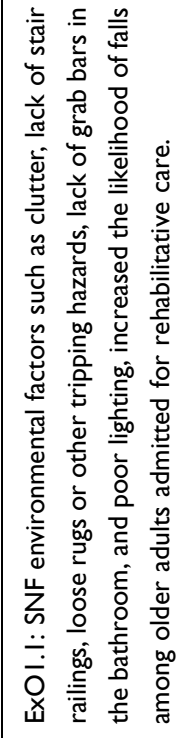 & 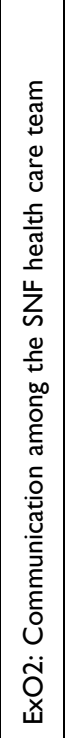 & 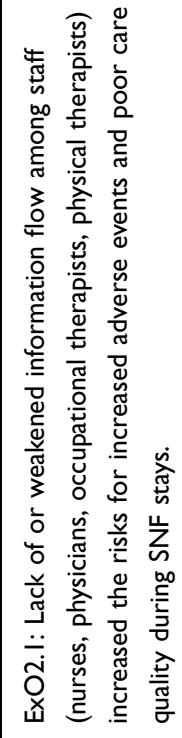 & 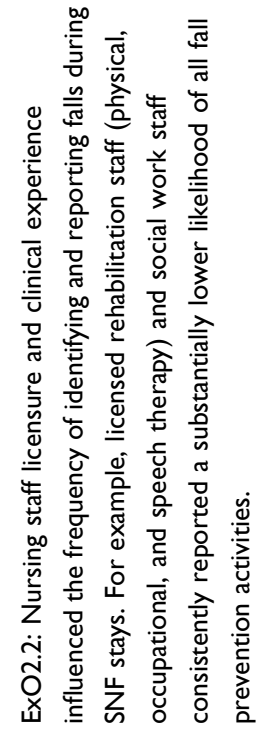 & 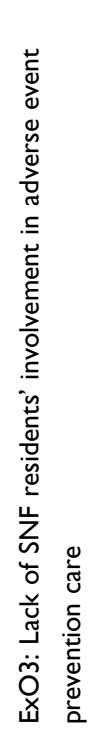 \\
\hline 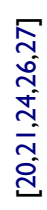 & 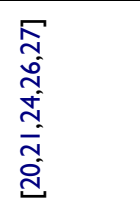 & $\begin{array}{l}\underset{J}{d} \\
\underset{d}{ }\end{array}$ & $\underset{\mathfrak{J}}{\mathbb{d}}$ & 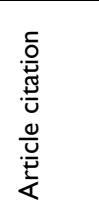 & 1 & & & & 1 & \\
\hline 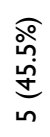 & 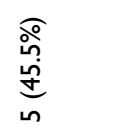 & $\begin{array}{l}\widehat{\circ} \\
\stackrel{\circ}{\circ} \\
\stackrel{0}{0} \\
\stackrel{+}{\sigma}\end{array}$ & $\begin{array}{l}\widehat{o} \\
\stackrel{+}{+} \\
\stackrel{0}{0} \\
\stackrel{0}{\sigma}\end{array}$ & 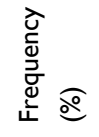 & 1 & & & & 1 & \\
\hline 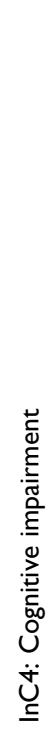 & 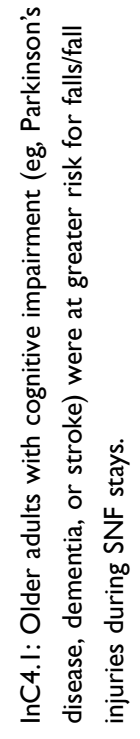 & 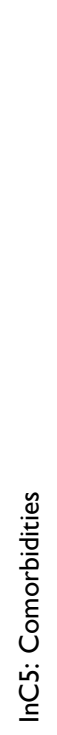 & 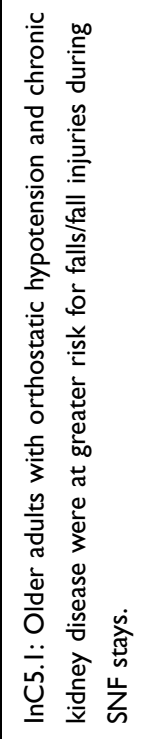 & 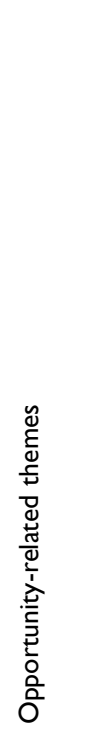 & 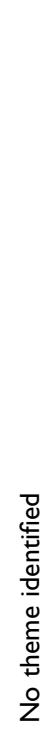 & & & & 1 & \\
\hline
\end{tabular}




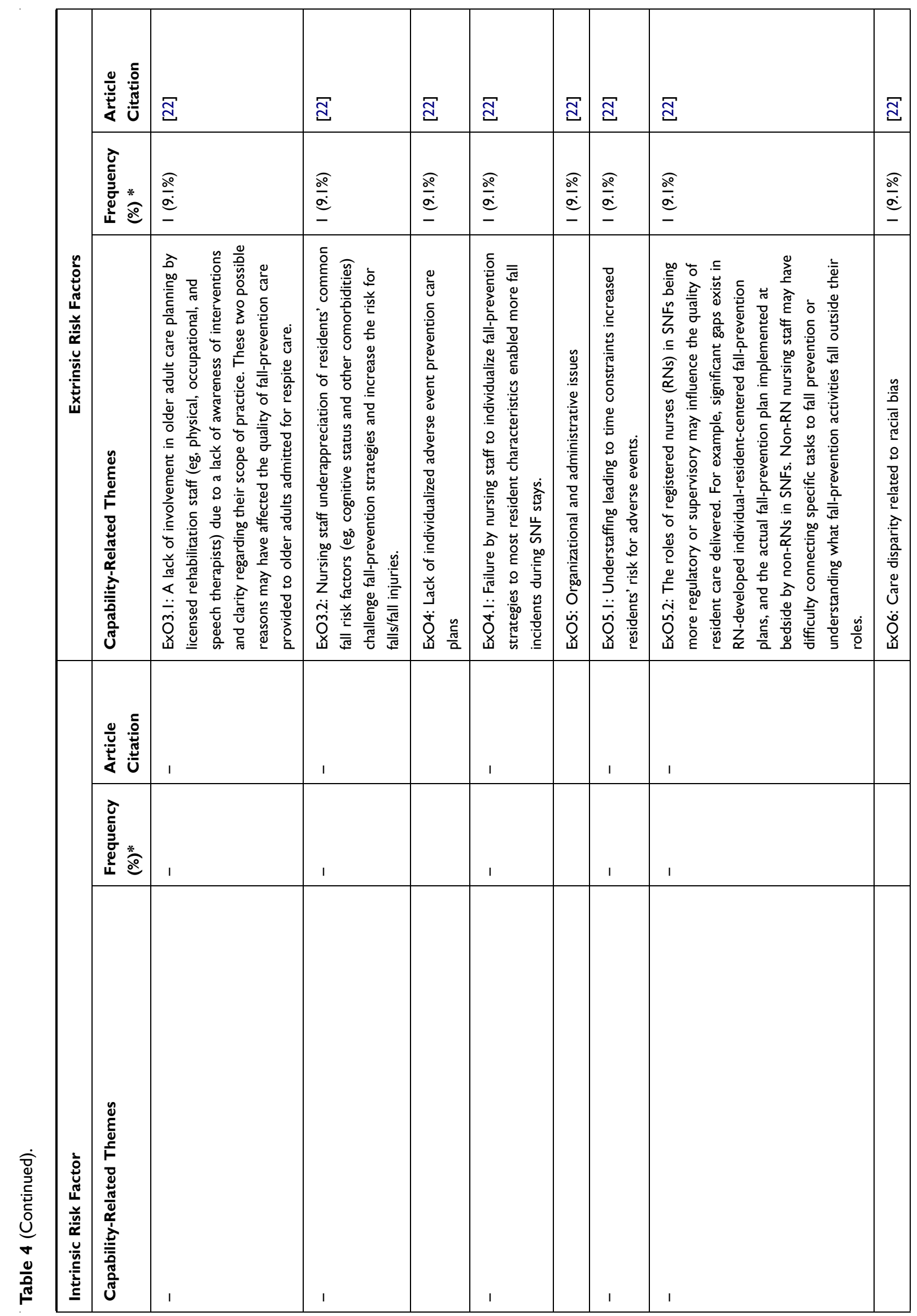




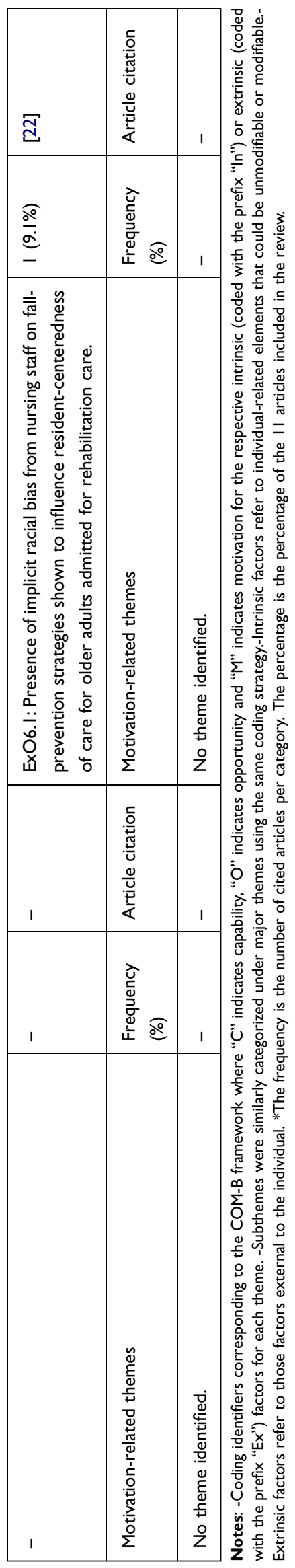

extrinsic contributors to adverse events in SNFs may help guide interventional strategies (capability-related elements) and opportunities for change (opportunity- and motivation-related elements) as health professionals navigate older adult care along the care continuum.

The contributors to adverse events among short-stay SNF residents identified herein were similar to those found for older adults participating in fall-prevention strategies after transitioning home from acute hospitalization. ${ }^{30}$ The present study validates the importance of identifying individualized resident care plans during short SNF stays for rehabilitation. These intrinsic factors were inevitably related and thus contributed to singular adverse event incidents, such as falls and medication errors, and contributed additively and synergistically to other adverse events. For example, capacity-related intrinsic factors, such as advancing age, coexisted with physical and cognitive disabilities in most articles on falls and fall injuries. ${ }^{6,20-28}$

This scoping review identified the risks of major medication classes, especially CNS-related drugs, to adverse events during short stays. ${ }^{20,21,23,28,29}$ The adverse effects of these medications (eg, sedation, impaired balance, delirium, and discoordination), as well as the drug interactions from taking multiple medications (polypharmacy), were identified as modifiable capacity-related extrinsic contributors to adverse events such as falls and fall injuries during short stays in SNFs. ${ }^{20,21,23,28,29}$ Studies substantiating polypharmacy and inappropriate medications as significant contributors to frequent falls in $\mathrm{SNFs}^{20,21,23,28,29}$ recommended medication reviews and modifications combined with continued nursing staff education as a valuable strategy to mitigate medication errors and drug-drug interactions causing adverse events in SNFs. ${ }^{31,32}$

This study identified only one article ${ }^{22}$ that provided several opportunity-related extrinsic factors that contributed to adverse events during older adults' short stays in SNFs. Notably, these organizational and administrative challenges spanned the older adults' continuum of care. ${ }^{22}$ Previous studies supported the importance of addressing communication and older adults' engagement in individualized care to improve their safety, prevent falls at home, enhance health quality, and promote self-care among community-dwelling older adults. ${ }^{10,30,33,34}$

\section{Practical Implications}

The findings of this scoping review may guide interventional strategies for mitigating adverse events among older adults during short SNF stays by addressing the intrinsic and 
extrinsic contributors. SNF administrators play vital roles in leading the efforts to decrease adverse events. The capacityrelated and opportunity-related extrinsic contributors identified herein (ie, poor communication among SNF staff regarding residents, lack of individualized care plans or resident engagement in the care plans, resident discrimination, and lack of institutional support for staff in SNFs) shed light on areas that warrant further research and interventions.

\section{Study Strengths and Limitations}

The main strength of this scoping review was that we applied the COM-B model of health behavioral change ${ }^{13}$ as a framework to identify and categorize contributing factors. By differentiating intrinsic versus extrinsic contributors, the COM-B model provided a novel approach to pursuing our study's research objectives and facilitating future interventional strategy development and evaluation. The COM-B behavioral change wheel program planning model $^{35}$ guides matching various intervention components with specific elements of the COM-B model. ${ }^{13}$

The study had some limitations. First, few peerreviewed sources met the eligibility criteria to enable us to develop our findings across all the domains of the COM-B framework. Second, the authors could not overtly ascertain whether all studies addressed only short-stay older adult residents. We repeatedly reached out to the corresponding authors of these studies but received responses from only two of them.

\section{Conclusion}

We reviewed the main findings of 11 original peerreviewed articles to present an overview of intrinsic and extrinsic contributors to adverse events among older adults admitted to SNFs for short stays. The review used the COM-B model of health behavioral change $^{13}$ to identify the contributors to adverse events (ie, falls, medication errors, pressure ulcers, and acute infections). Intrinsic and extrinsic factors leading to adverse events were grouped across each capabilityopportunity-motivation domain related to health behavioral changes among older adults during short stays for rehabilitation. Capacity-related intrinsic contributors to adverse events were frailty, reduced muscle strength due to advancing age and history of falls. Capacity-related extrinsic factors identified were inappropriate medications and issues related to polypharmacy, and opportunity-related extrinsic contributors identified included environmental hazards, poor communication among SNF staff, lack of individualized care plans, lack of resident engagement in the care plans, resident discrimination, and lack of institutional support for staff in SNFs. We believe our findings could help shed light on areas that would warrant further research and guide interventional strategies for mitigating adverse events among older adults during short SNF stays.

\section{Disclosure}

The authors report no conflicts of interest in this work.

\section{References}

1. U.S. Department of Health and Human Services Office of the Inspector General. Adverse events in skilled nursing facilities: national incidence among medicare beneficiaries; 2014. Available from: https://oig.hhs.gov/oei/reports/oei-06-11-00370.pdf. Accessed October 19, 2021.

2. Agency for Healthcare Research and Quality (AHRQ). Adverse events, near misses, and errors; 2019. Available from: https://psnet. ahrq.gov/primer/adverse-events-near-misses-and-errors. Accessed October 19, 2021.

3. Kapoor A, Field T, Handler S, et al. Adverse events in long-term care residents transitioning from hospital back to nursing home. JAMA Intern Med. 2019;179(9):1254-1261. doi:10.1001/ jamainternmed.2019.2005

4. Kapoor A, Field T, Handler S, et al. Characteristics of long-term care residents that predict adverse events after hospitalization. $\mathrm{J} \mathrm{Am}$ Geriatr Soc. 2020;68(11):2551-2557. doi:10.1111/jgs.16770

5. Da Costa-dias MJM, Lopes Ferreira P. Fall risk assessment tools. Revista De Enfermagem Referência. 2014;4(2):153-161.

6. Datta A, Datta R, Elkins J. What factors predict falls in older adults living in nursing homes: a Pilot Study. J Funct Morphol Kinesiol. 2018;4(1):3-10. doi:10.3390/jfmk4010003

7. Khow KSF, Visvanathan R. Falls in the aging population. Clin Geriatr Med. 2017;33(3):357-368. doi:10.1016/j.cger.2017.03.002

8. Simmons S, Schnelle J, Slagle J, et al. AHRQ comparative effectiveness technical briefs. In: Resident Safety Practices in Nursing Home Settings. Rockville (MD): Agency for Healthcare Research and Quality (US); 2016.

9. Hakkarainen TW, Arbabi S, Willis MM, Davidson GH, Flum DR. Outcomes of patients discharged to skilled nursing facilities after acute care hospitalizations. Ann Surg. 2016;263(2):280-285. doi:10.1097/SLA.0000000000001367

10. Tzeng HM, Jansen LS, Okpalauwaekwe U, Khasnabish S, Andreas B, Dykes PC. Adopting the fall Tailoring Interventions for Patient Safety (TIPS) program to engage older adults in fall prevention in a nursing home. J Nurs Care Qual. 2021;36(4):327-332. doi:10.1097/NCQ.0000000000000547

11. Center for Disease Control and Prevention (CDC). Fall data: cost of older adult falls; 2019. Available from: https://www.cdc.gov/ HomeandRecreationalSafety/Falls/fallcost.html. Accessed May 23, 2021.

12. Milat AJ, Watson WL, Monger C, Barr M, Giffin M, Reid M. Prevalence, circumstances and consequences of falls among community-dwelling older people: results of the 2009 NSW falls prevention baseline survey. N S W Public Health Bull. 2011;22(34):43-48. doi:10.1071/NB10065

13. Michie S, van Stralen MM, West R. The behaviour change wheel: a new method for characterising and designing behaviour change interventions. Implement Sci. 2011;6:42. doi:10.1186/1748-5908-6-42 
14. Peters MD, Godfrey CM, Khalil H, McInerney P, Parker D, Soares CB. Guidance for conducting systematic scoping reviews. Int $J$ Evid Based Healthc. 2015;13(3):141-146. doi:10.1097/ XEB.0000000000000050

15. Arksey H, O’Malley L. Scoping studies: towards a methodological framework. Int J Soc Res Methodol. 2005;8(1):19-32. doi:10.1080/ 1364557032000119616

16. Tricco AC, Lillie E, Zarin W, et al. PRISMA extension for scoping reviews (PRISMA-ScR): checklist and explanation. Ann Intern Med. 2018;169(7):467-473. doi:10.7326/M18-0850

17. MacOS Commercial Software [Computer Program]. Thomson Reuters Corporation [computer program]; 2019.

18. Microsoft excel version 16.32 [Computer Program]; 2016.

19. Joanna Briggs Institute. Joanna Briggs Institute critical appraisal tools; 2014. Available from: https://joannabriggs.org/ebp/critical_ appraisal tools. Accessed May 23, 2021.

20. Aspinall SL, Springer SP, Zhao X, et al. Central nervous system medication burden and risk of recurrent serious falls and hip fractures in veterans affairs nursing home residents. J Am Geriatr Soc. 2019;67 (1):74-80. doi:10.1111/jgs.15603

21. Aspinall SL, Zhao X, Semla TP, et al. Epidemiology of drug-disease interactions in older veteran nursing home residents. $J$ Am Geriatr Soc. 2015;63(1):77-84. doi:10.1111/jgs.13197

22. Colón-Emeric CS, Corazzini K, McConnell E, et al. Study of individualization and bias in nursing home fall prevention practices. $\mathrm{J} \mathrm{Am}$ Geriatr Soc. 2017;65(4):815-821. doi:10.1111/jgs.14675

23. Cox CA, van Jaarsveld HJ, Houterman S, et al. Psychotropic drug prescription and the risk of falls in nursing home residents. $J \mathrm{Am} \mathrm{Med}$ Dir Assoc. 2016;17(12):1089-1093. doi:10.1016/j.jamda.2016.07.004

24. Hall RK, Landerman LR, O'Hare AM, Anderson RA, Colón-Emeric CS. Chronic kidney disease and recurrent falls in nursing home residents: a retrospective cohort study. Geriatr Nurs. 2015;36(2):136-141. doi:10.1016/j.gerinurse.2014.12.012

25. Bell SP, Vasilevskis EE, Saraf AA, et al. Geriatric syndromes in hospitalized older adults discharged to skilled nursing facilities. J Am Geriatr Soc. 2016;64(4):715-722. doi:10.1111/jgs.14035

26. Hartog LC, Cimzar-Sweelssen M, Knipscheer A, et al. Orthostatic hypotension does not predict recurrent falling in a nursing home population. Arch Gerontol Geriatr. 2017;68:39-43. doi:10.1016/j. archger.2016.08.011
27. Hartog LC, Cizmar-Sweelssen M, Knipscheer A, et al. The association between orthostatic hypotension, falling and successful rehabilitation in a nursing home population. Arch Gerontol Geriatr. 2015;61 (2):190-196. doi:10.1016/j.archger.2015.05.005

28. Bronskill SE, Campitelli MA, Iaboni A, et al. Low-dose trazodone, benzodiazepines, and fall-related injuries in nursing homes: a Matched-Cohort Study. J Am Geriatr Soc. 2018;66(10):1963-1971. doi:10.1111/jgs.15519

29. Nyborg G, Brekke M, Straand J, Gjelstad S, Romøren M. Potentially inappropriate medication use in nursing homes: an observational study using the NORGEP-NH criteria. BMC Geriatr. 2017;17 (1):220. doi:10.1186/s12877-017-0608-z

30. Tzeng HM, Okpalauwaekwe U, Lyons EJ. Barriers and facilitators to older adults participating in fall-prevention strategies after transitioning home from acute hospitalization: a scoping review. Clin Interv Aging. 2020;15:971-989. doi:10.2147/CIA.S256599

31. Pit SW, Byles JE, Henry DA, Holt L, Hansen V, Bowman DA. A quality use of medicines program for general practitioners and older people: a cluster randomised controlled trial. Med J Aust. 2007;187(1):23-30. doi:10.5694/j.1326-5377.2007.tb01110.x

32. Enderlin C, Rooker J, Ball S, et al. Summary of factors contributing to falls in older adults and nursing implications. Geriatr Nurs. 2015;36(5):397-406. doi:10.1016/j.gerinurse.2015.08.006

33. Tzeng HM, Okpalauwaekwe U, Li CY. Older adults' sociodemographic determinants of health related to promoting health and getting preventive health care in southern United States: a secondary analysis of a survey project dataSET. Nurs Rep. 2021;11(1):120-132. doi:10.3390/nursrep 11010012

34. Tzeng HM, Okpalauwaekwe U, Feng C, Jansen SL, Barker A, Yin CY. Exploring associations between older adults' demographic characteristics and their perceptions of self-care actions for communicating with healthcare professionals in southern United States. Nurs Open. 2019;6(3):1133-1142. doi:10.1002/nop2.315

35. Michie SA, Atkins L, West R. The behaviour change wheel: a guide to designing interventions: the COM-B self-evaluation questionnaire can be found Pp. 68-69.[Permission granted by Dr. Michie to use this questionnaire]. Great Britain: Silverback Publishing; 2014.
Patient Related Outcome Measures

\section{Publish your work in this journal}

Patient Related Outcome Measures is an international, peer-reviewed, open access journal focusing on treatment outcomes specifically relevant to patients. All aspects of patient care are addressed within the journal and practitioners from all disciplines are invited to submit their work as well as healthcare researchers and patient support groups.
The manuscript management system is completely online and includes a very quick and fair peer-review system. Visit http://www. dovepress.com/testimonials.php to read real quotes from published authors. 